\title{
THE RESIDUE THEOREM AND AN ANALOG OF P. APPELL'S FORMULA FOR FINITE RIEMANN SURFACES
}

\author{
Viktor V. Chueshev*, Aleksandr V. Chueshev
}

Kemerovo State University, Krasnaya Str. 6, Kemerovo, 650043 Russian Federation

Received April 19, 2016;

* e-mail: vvchueshev@ngs.ru

Accepted in revised form June 10, 2016;

Published June 30, 2016

\begin{abstract}
A theory of multiplicative functions and Prym differentials for the case of special characters on compact Riemann surfaces has found applications in geometrical function theory of complex variable, analytical number theory and in equations of mathematical physics. Theory of functions on compact Riemann surfaces differs from the theory of functions on finite Riemann surfaces even for the class of single meromorphic functions and Abelian differentials. In this article we continue the construction of the general function theory on finite Riemann surfaces for multiplicative meromorphic functions and differentials. We have proved analogues of the theorem on the full sum of residues for Prym differentials of every integral order and P. Appell's formula on expansion of the multiplicative function with poles of arbitrary multiplicity in the sum of elementary Prym integrals.
\end{abstract}

Keywords: Teichmuller spaces for finite Riemann surfaces, Prym differentials, group of characters, Jacobi manifolds

\section{INTRODUCTION}

Theory of multiplicative functions and Prym differentials for the case of special characters on compact Riemann surface has found applications in geometrical function theory of complex variable, analytical number theory and in equations of mathematical physics [1-7]. In [2, 4] the construction of the general theory of multiplicative functions and Prym differentials on compact Riemann surface for arbitrary characters was started. Theory of functions on compact Riemann surfaces differs from the theory of functions on finite Riemann surfaces even for the class of single meromorphic functions and Abelian differentials. A series of general spaces of functions and differentials on finite Riemann surface $F^{\prime}$ of genus ( $g, n), g \geq 1, n>0$ will be infinite-dimensional.

In this article we continue the construction of the general function theory on finite Riemann surfaces for multiplicative meromorphic functions and differentials. Analogues of the theorem on the full sum of residues for Prym differentials of every integral order and P. Appell's formula on expansion of the multiplicative function with poles of arbitrary multiplicity in the sum of elementary Prym integrals have been proved.

\section{MATERIALS AND METHODS 1. Preliminaries}

Let $F$ be a fixed smooth compact oriented surface of genus $g \geq 2$ with $\left\{a_{k}, b_{k}\right\}_{k=1}^{g}$, and $F_{0}$ be a compact
Riemann surface with fixed complex analytic structure on $F$. Let us fix different points $P_{1}, \ldots, P_{n} \in F$. Let $F^{\prime}=F \backslash\left\{P_{1}, \ldots, P_{n}\right\} \quad$ be a surface of type $(g, n), n \geq 1, g \geq 2$. Any other structure on $F^{\prime}$ is given by some Bertrami differential $\mu$ on $F_{0}^{\prime}$, i.e. by the expression of the form $\mu(z) \frac{d \bar{z}}{d z}$, which is invariant relative to the choice of the local parameter on $F_{0}^{\prime}$, where $\mu(z)$ is complex-valued function on $F_{0}^{\prime}$ and $|\mu|_{L_{\infty}\left(F_{0}^{\prime}\right)}<1$. This structure on $F^{\prime}$ we will denote by $F_{\mu}^{\prime}$. It is uniformized by quasi-Fuchsian group $\Gamma_{\mu}^{\prime}$.

In the work of L. Bers [3] Abelian differentials $\zeta_{1}[\mu], \ldots, \zeta_{g}[\mu]$ on $F_{\mu}$, which form a canonical basis dual to the canonical homotopy basis $\left\{a_{k}^{\mu}, b_{k}^{\mu}\right\}_{k=1}^{g}$ on $F_{\mu}$ have been constructed and also it holomorphically depends on points $[\mu]$ of the Teichmuller space $T_{g}$. Moreover, the matrix of $b$-periods $\Omega(\mu)=\left(\pi_{j k}[\mu]\right)_{j, k=1}^{g}$ on $F_{\mu} \quad$ consists of complex numbers $\left(\pi_{j k}[\mu]\right)=\int_{\xi}^{B_{k}^{\mu}(\xi)} \zeta_{j}([\mu], w) d w, \xi \in w^{\mu}(U)$, and holomorphically depends on $[\mu]$.

For any fixed $[\mu] \in T_{g}$ and $\xi_{0} \in w^{\mu}(U)$ let us define a classical Jacobi mapping $\varphi: w^{\mu}(U) \rightarrow C^{g}$

Please cite this article in press as: Chueshev V.V., Chueshev A.V. The residue theorem and an analog of P. Appell's formula for finite Riemann surfaces. Science Evolution, 2016, vol. 1, no. 1, pp. 40-45.

Copyright (C) 2016, KemSU. This is an open access article distributed under the terms of the Creative Commons Attribution 4.0 International License (http://creativecommons.org/licenses/by/4.0/), allowing third parties to copy and redistribute the material in any medium or format and to remix, transform, and build upon the material for any purpose, even commercially, provided the original work is properly cited and states its license. This article is published with open access at http://science-evolution.ru/. 
according to the rule $\varphi_{j}(\xi)=\int_{\xi_{0}}^{\xi} \zeta_{j}([\mu], w) d w$, $j=1, \ldots, g$.

A quotient space $J(F)=C^{g} / L(F)$ is called marked Jacobi manifold for $F=F_{0}$, where $L(F)$ is a lattice above $Z$, generated by columns $e^{(1)}, \ldots, e^{(g)}, \pi^{(1)}, \ldots, \pi^{(g)}$ of the matrix $\left(I_{g}, \Omega\right)[6,7]$.

Any homomorphism $\rho:\left(\pi_{1}\left(F_{\mu}^{\prime}\right),.\right) \rightarrow\left(C^{*},.\right), C^{*}=$ $=C \backslash\{0\}$ is called the character $\rho$ for $F_{\mu}^{\prime}$. Further we will assume that $\rho\left(\gamma_{j}^{\mu}\right)=1$, where $\gamma_{j}^{\mu}$ is a simple loop that avoid puncture $P_{j}$ on $F_{\mu}^{\prime}, j=1, \ldots, n$.

Definition 1.1. Meromorphic function $f$ on $w^{\mu}(U)$ is called a multiplicative function $f$ on $F_{\mu}^{\prime}$ for the character $\rho$ if $f(T z)=\rho(T) f(z)$, $z \in w^{\mu}(U), T \in \Gamma_{\mu}^{\prime}$.

Definition 1.2. Differential $\omega(z) d z^{q}$ is called $q$-Prym differential in relation to Fuchsian group $\Gamma_{\mu}^{\prime}$ for $\rho$, or $(\rho, q)$ - differential, if $\omega(T z)\left(T^{\prime} z\right)^{q}=$ $=\rho(T) \omega(z), \quad z \in U, T \in \Gamma^{\prime}, \rho: \Gamma^{\prime} \rightarrow C^{*}$

If $f_{0}$ is a multiplicative function on $F_{\mu}$ for $\rho$ without zeros or poles, then $\rho$ characters for such functions we shall call inessential and $f_{0}$ we shall call a unit. Characters that are not inessential we shall call essential on $\pi_{1}\left(F^{\mu}\right)$. A set $L_{g}$ of inessential characters forms a subgroup in the group $\operatorname{Hom}\left(\Gamma, C^{*}\right)$ of all characters on $\Gamma$.

Theorem (of Abel for characters) [2, 4]. Let $D=P_{1}^{n_{1}} \ldots P_{k}^{n_{k}}, P_{j} \in F_{\mu}, n_{j} \in Z, j=1, \ldots, k$, be a divisor on a marked variable compact Riemann surface $\left[F_{\mu}, a_{1}^{\mu}, \ldots, a_{g}^{\mu}, b_{1}^{\mu}, \ldots, b_{g}^{\mu}\right]$ of genus $g \geq 1$, and let $\rho$ be a character on $\pi_{1}\left(F_{\mu}\right)$. Then $D$ will be a divisor of the function $f$ on $F_{\mu}$ for $\rho \Leftrightarrow \operatorname{deg} D=0$ and $\varphi(D)=\frac{1}{2 \pi i} \sum_{j=1}^{g} \log \rho\left(b_{j}^{\mu}\right) e^{(j)}[\mu] \quad-\log \rho\left(a_{j}^{\mu}\right) \pi^{(j)}[\mu] \equiv$ $\equiv \psi(\rho,[\mu])$, where $\varphi[\mu]: F_{\mu} \rightarrow J\left(F_{\mu}\right) \quad$ is a Jacobi mapping.

Class $M_{1}(\rho)$ consists of Prym differentials for $\rho$ on $F_{\mu}^{\prime}$ which have a finite number of poles on $F_{\mu}^{\prime}$ and allow meromorphic continuation on $F_{\mu}$.

In $[6,7]$ it was proved that for any essential character $\rho$, points $Q_{1} \in F_{\mu}$, of natural number $q \geq 1$ and inessential character $\rho$, points $Q_{1} \in F_{\mu}$, of natural number $q>1$ exists elementary $(\rho, q)$ - differential $\tau_{\rho, q ; Q_{1}}$ of third kind with a single simple pole $Q_{1}[\mu]$ on $F_{\mu}$. For any inessential character $\rho$, points $Q_{1} \in F_{\mu}$, when $q=1$, elementary $(\rho, 1)$-differential $\tau_{\rho, q ; Q_{1}}$ doesn't exist. There it was also proved that on a variable surface $F_{\mu}$ of kind $g \geq 2$ for any natural number $q \geq 1$ exists elementary $(\rho, q)$-differential $\tau_{\rho, q ; Q_{1}, Q_{2}}$ of third kind with simple poles $Q_{1}, Q_{2} \in F_{\mu}$, and $\tau_{\rho, q ; Q_{1}}^{(m)}=\left(\frac{1}{z^{m}}+O(1)\right) d z^{q}$ of second genus with the pole $Q_{1}[\mu]$ of order $m \geq 2$. These differentials locally holomorphically depend on $[\mu]$ and $\rho$.

\section{An analogue of the residue theorem for Prym differentials on finite Riemann surface}

Residues for Prym differentials can be defined only for the branches of these multivalued differentials.

Let $\tau$-be a $(\rho, q)-$ differential such that $(\tau) \geq \frac{1}{Q_{1}^{\alpha_{1}} \ldots Q_{s}^{\alpha_{s}}}, \quad s \geq 1, \alpha_{j} \geq 0, \alpha_{j} \in N, j=1, \ldots, s$, with pairwise distinct points $Q_{1}, \ldots, Q_{s}$ on $F^{\prime}$.

Analytic continuation $\tau$ (hereinafter referred to with this symbol) with $F^{\prime}$ on $F$, meets conditions

$$
(\tau) \geq \frac{1}{Q_{1}^{\alpha_{1}} \ldots Q_{s}^{\alpha_{s}} P_{1}^{k_{1}} \ldots P_{n}^{k_{n}}}, k_{j} \geq 0, k_{j} \in N, j=1, \ldots, n .
$$

Let us introduce the following notations:

1) if $\rho$ is inessential character, then let us choose multiplicative unit on $F$ for $\rho^{-1}$, where

$$
\begin{gathered}
f_{0}=\exp \left[-\int_{Q_{0}}^{P} \sum_{j=1}^{g} \log \rho\left(a_{j}\right) \zeta_{j}\right], \\
Q_{0} \neq Q_{1}, \ldots, Q_{s}, P_{1}, \ldots, P_{n} ;
\end{gathered}
$$

2) if $\rho$ is an essential character, then there exists a single function $f_{1}$ on $F$ for $\rho^{-1}$ with a single simple pole $P_{1}$ [6]. Such function $f_{1}$ has a divisor $\frac{R_{1}}{P_{1}}$, where $\varphi\left(R_{1}\right)=\varphi\left(P_{1}\right)-\psi(\rho) \quad$ in Jacobi manifold $J(F), \psi(\rho) \neq 0$. This function may be presented in a form of

$$
\begin{gathered}
f_{1}(P)=\exp \left[\int_{Q_{0}}^{P}\left(\tau_{R_{1} P_{1}}-\sum_{j=1}^{g} \log \rho\left(a_{j}\right) \zeta_{j}\right)\right], \\
Q_{0} \neq Q_{1}, \ldots, Q_{s}, P_{1}, \ldots, P_{n}
\end{gathered}
$$

Let us show uniqueness of such function. If there is a point $R_{2}$ such that the equality $\varphi\left(R_{1}\right)=\varphi\left(P_{1}\right)-\psi(\rho)=\varphi\left(R_{2}\right) \quad$ is true, then $\varphi\left(\frac{R_{1}}{R_{2}}\right)=0$. According to classical Abel's theorem there is a single-valued function $h$ with the divisor $(h)=\frac{R_{1}}{R_{2}}$ that has a single simple pole on compact Riemann surface of a positive genus. A contradiction.

Without loss of generality we can find Abelian holomorphic 1-differential $\omega_{0}$ such that $\left(\omega_{0}\right) \cap\left\{Q_{1}, \ldots, Q_{s}, P_{1}, \ldots, P_{n}\right\}=\varnothing \quad$ on $\quad F$, because 
divisors of Abelian holomorphic differentials don't have base points on $F$ [4]. Let us choose any Abelian differential $\omega_{0}$ with the divisor $\left(\omega_{0}\right)=S_{1}, \ldots, S_{2 g-2}$ on $F$, so that there were as few points as possible in its divisor. We have an equality $\varphi_{P_{0}}\left(S_{1} \ldots S_{2 g-2}\right)=-2 K$ in Jacobi manifold $J(F)$, where $K$ is a vector of Riemann constant for a marked compact Riemann surface $F$ with the base point $P_{0}$ [4]. This equality is equivalent to another equality in the form $\varphi_{S_{g+1}}\left(S_{1} \ldots S_{g}\right)=-2 K-\varphi_{S_{g+1}}\left(S_{g+1}^{g-2}\right), \quad$ where $P_{0}=S_{g+1}=\ldots=S_{2 g-2}$. This implies Abelian differential $\omega_{0}$ with divisor in the form $\left(\omega_{0}\right)=S_{1} \ldots S_{g} S_{g+1}^{g-2}$.

Let us make Abelian 1-differentials $\frac{\tau f_{0}}{\omega_{0}^{q-1}}$ and $\frac{\tau f_{1}}{\omega_{0}^{q-1}}$ on $F$, where $f_{0}$ and $f_{1}$ have character $\rho^{-1}$ on $F$ and differential $\tau$ is analytically continued from $F^{\prime}$ on $F$. By the theorem on a complete sum of residues for Abelian 1-differentials $\frac{\tau f_{j}}{\omega_{0}^{q-1}}, j=0,1$, on $F$ we obtain the following analogue of the theorem on a complete sum of residues for $(\rho, q)$ - differentials.

Theorem 2.1.

1) For any $(\rho, q)$ - differential $\tau$ of the class $M_{1}$ on Riemann surface $F$ of type $(g, n), g \geq 2, n \geq 1$, with any polar divisor $(\tau)_{\infty}=Q_{1}^{\alpha_{1}} \ldots Q_{s}^{\alpha_{s}}$, of any integer $q$ and unit $f_{0}$ for inessential character $\rho^{-1}$ on $F$ the following equality is true:

$$
\begin{aligned}
& \sum_{j=1}^{s} \operatorname{res}_{Q_{j}} \frac{\tau f_{0}}{\omega_{0}^{q-1}}+\sum_{j=1}^{g+1} \operatorname{res}_{S_{j}} \frac{\tau f_{0}}{\omega_{0}^{q-1}}+ \\
& +\sum_{j=1}^{n} \operatorname{res}_{P_{j}} \frac{\tau f_{0}}{\omega_{0}^{q-1}}=0
\end{aligned}
$$

2) For any $(\rho, q)-$ differential $\tau$ of the class $M_{1}$ on Riemann surface $F$ of type $(g, n), g \geq 2, n \geq 1$, with any polar divisor $(\tau)_{\infty}=Q_{1}^{\alpha_{1}} \ldots Q_{s}^{\alpha_{s}}$, of any integer $q$ and single, accurate to multiplication by a nonzero constant, function $f_{1}$ for essential character $\rho^{-1},\left(f_{1}\right) \geq \frac{1}{P_{1}}$ on $F$ the following equality is true:

$$
\begin{gathered}
\sum_{j=1}^{s} \operatorname{res}_{Q_{j}} \frac{\tau f_{1}}{\omega_{0}^{q-1}}+\sum_{j=1}^{g+1} \operatorname{res}_{S_{j}} \frac{\tau f_{1}}{\omega_{0}^{q-1}}+ \\
+\sum_{j=1}^{n} \operatorname{res}_{P_{j}} \frac{\tau f_{1}}{\omega_{0}^{q-1}}=0 .
\end{gathered}
$$

In both cases $\omega_{0}$ is an Abelian holomorphic differential with a divisor

$$
\left(\omega_{0}\right)=S_{1} \ldots S_{g} S_{g+1}^{g-2}, \varphi_{S_{g+1}}\left(S_{1} \ldots S_{g}\right)=-2 K
$$

in Jacobi manifold $J(F)$ and $\left\{S_{1}, \ldots, S_{g}, S_{g+1}\right\} \cap\left\{Q_{1}, \ldots, Q_{s}, P_{1}, \ldots, P_{n}\right\}=\varnothing$ on $F$.

Remark 2.1. Note that in the preceding theorem when $q \leq 0$ there is no second sum in the assertion of the theorem.

Remark 2.2. P. Appell considered the residue theorem only when $q=0$ on compact Riemann surface of kind $g>1$.

Let us find some corollaries of the residue theorem and reciprocity laws for multiplicative functions on finite Riemann surface. First we find corollary for 1differential $\omega$ with any character $\rho$, in a special case, when $\omega=d f$, where $f$ is a multiplicative function on $F^{\prime}$ of the $M_{1}$ class.

Let $R_{1}, \ldots, R_{m}$ be zeros of $f$ with multiplicity $\lambda_{1}, \ldots, \lambda_{m}$ and let $Q_{1}, \ldots, Q_{s}$ be poles for $f$ with multiplicity $\mu_{1}, \ldots ., \mu_{s}$ when the function $f$ is continued analytically from $F^{\prime}$ to $F$. Let us also consider single-valued function $h$ on $F$ with poles $L_{1}, \ldots, L_{l}$ of multiplicity $p_{1}, \ldots, p_{l}$ accordingly where points $L_{i}$ are not included in the support of the divisor $\sup p(f)$. Let us note that $\frac{d f}{f}$ will be an Abelian differential with simple poles $R_{1}, \ldots, R_{m}, Q_{1}, \ldots, Q_{s}$ and residues $\lambda_{1}, \ldots, \lambda_{m},-\mu_{1}, \ldots,-\mu_{s}$ in them accordingly. Then, because of uniqueness of the expression under integral and by the residue theorem for Abelian 1-differentials, we obtain that:

$$
\begin{gathered}
0=\frac{1}{2 \pi i} \int_{\partial \Delta_{\mu}} h \frac{d f}{f}=\sum_{k=1}^{m} \lambda_{k} h\left(R_{k}\right)- \\
-\sum_{j=1}^{s} \mu_{k} h\left(Q_{j}\right)+\sum_{i=1}^{l} \operatorname{res}_{L_{i}} h \frac{d f}{f},
\end{gathered}
$$

where $\Delta$ is a connected fundamental domain for group $\Gamma$ in domain $U[3,5]$.

From (1) we will obtain formulas that are connected with a special choice of a function $h$ on $F$ :

1) Let $h$ be an analytical function on $F$, and $h$ be a constant. Then $\sum_{k=1}^{m} \lambda_{k}=\sum_{j=1}^{s} \mu_{j}$ is a classical fact that $\operatorname{deg}(f)=0$ on $F$ [4];

2) If $h$ has multiple poles in points $L_{i}$, i.e.

$$
h=\frac{c_{-p_{i}}^{(i)}}{\left(z-z\left(L_{i}\right)\right)^{p_{i}}}+\ldots+\frac{c_{-1}^{(i)}}{z-z\left(L_{i}\right)}+O(1), i=1, \ldots, l,
$$

then we obtain the equality

$$
\begin{aligned}
& \sum_{j=1}^{s} \mu_{j} h\left(Q_{j}\right)-\sum_{k=1}^{m} \lambda_{k} h\left(R_{k}\right)= \\
& =\sum_{i=1}^{l}\left[\left(\frac{f^{\prime}}{f}\right)^{\left(p_{i}-1\right)}\left(L_{i}\right) \frac{c_{-p_{i}}^{(i)}}{\left(p_{i}-1\right) !}+\left(\frac{f^{\prime}}{f}\right)^{\left(p_{i}-2\right)}\left(L_{i}\right) \frac{c_{-p_{i}+1}^{(i)}}{\left(p_{i}-2\right) !}+\ldots+\left(\frac{f^{\prime}}{f}\right)^{\prime}\left(L_{i}\right) c_{-2}^{(i)}+\left(\frac{f^{\prime}}{f}\right)\left(L_{i}\right) c_{-1}^{(i)}\right] .
\end{aligned}
$$


Remark 2.3. These two equalities are some reciprocity laws that connect zeros and poles for the multiplicative function $f$ of the class $M_{1}$ on $F^{\prime}$ with poles of single-valued meromorphic functions $h$ on $F$.

\section{An analogue of Appell's formula for a} multiplicative function expansion on a variable finite Riemann surface.

Let us denote through $T_{\rho ; Q}^{(1)}(z)=-\int \tau_{\rho ; Q}^{(2)}$ an elementary Prym integral of the second kind on $F_{\mu}$ for essential character $\rho$ with a single simple pole in $Q$ and residue +1 in $Q$ which holomorphically depends on $[\mu]$ and $\rho$, where $\tau_{\rho ; Q}^{(2)}$ has a zero residue in the point $Q$.

Let $f$ be a function on $F_{\mu}^{\prime}$ of the class $M_{1}$ for essential character $\rho$ with $S$ simple poles $P_{n+1}, P_{n+2}, \ldots, P_{n+s}$ and residues $c_{n+1}, \ldots, c_{n+s}$ in them accordingly for one of its branches. Let us take analytical continuation of this function (and denote it with the same symbol) $f$ with $F_{\mu}^{\prime}$ on $F_{\mu}^{\prime}$. Let us consider the expression

$$
f_{1}=f-c_{n+1} T_{\rho ; P_{n+1}}^{(1)}-\ldots-c_{n+s} T_{\rho ; P_{n+s}}^{(1)}-\sum_{j=1}^{g-1} \tilde{c}_{j} \int \tilde{\zeta}_{j},
$$

where $\tilde{c}_{j} \in C, j=1, \ldots, g-1$, and $\tilde{\zeta}_{1}, \ldots, \tilde{\zeta}_{g-1}$ are the basis of Prym differentials of the first kind for essential character $\rho$ on $F_{\mu}$, that holomorphically depends on $[\mu]$ and $\rho$ [2]. Then $f_{1}$ is a meromorphic singlevalued branch of the Prym integral with essential character $\rho$ on fundamental polygon $\Delta_{\mu}$ with divisor $\left(f_{1}\right) \geq \frac{1}{P_{1}^{k_{1}} \ldots P_{n}^{k_{n}}}, \quad k_{j} \geq 0, j=1, \ldots, n$, on $F_{\mu}$.

Among other things, Prym integral $f_{1}$ for $\rho$ has a branch whose principal parts of Laurent series match with principal parts of Laurent series in points $P_{j}, j=1, \ldots, n$, for $f$ and zeros $a_{m}$ are periods, $m=1, \ldots, g-1$, on $F_{\mu}$ [2]. Therefore

$$
f=\sum_{j=1}^{s} c_{n+j} T_{\rho ; P_{n+j}}^{(1)}+\sum_{j=1}^{g-1} \tilde{c}_{j} \int \tilde{\zeta}_{j}+f_{1} .
$$

If $P_{n+1}$ is a pole of $k_{n+1}, k_{n+1} \geq 2$ order, then in the preceding formula a summand $c_{n+1} T_{\rho ; P_{n+1}}^{(1)}$ should be replaced with the sum of the form

$$
\begin{gathered}
A_{n+1,1} T_{\rho ; P_{n+1}}^{(1)}+A_{n+1,2} \frac{\partial T_{\rho ; P_{n+1}}^{(1)}}{\partial P_{n+1}}+ \\
+\frac{A_{n+1,3}}{2} \frac{\partial^{2} T_{\rho ; P_{n+1}}^{(1)}}{\partial P_{n+1}^{2}}+\ldots+\frac{A_{n+1, k_{n+1}}}{\left(k_{n+1}-1\right) !} \frac{\partial^{k_{n+1}-1} T_{\rho ; P_{n+1}}^{(1)}}{\partial P_{n+1}^{k_{n+1}-1}},
\end{gathered}
$$

where $A_{n+1,1}$ are coefficients in the principal part of Laurent series for some branch of the function $f$ in the point $P_{n+1}, j=1, \ldots, k_{n+1}\left(P_{n+1}\right)$. Indeed, in the neighborhood of the point $P_{n+k}$ we have expansion

$$
\begin{gathered}
T_{\rho ; P_{n+k}}^{(1)}=\frac{1}{\left(z-z\left(P_{n+k}\right)\right)}+O(1) ;\left(T_{\rho ; P_{n+k}}^{(1)}\right)_{a_{k}}^{\prime}= \\
=\frac{1}{\left(z-a_{k}\right)^{2}}+O(1), z\left(P_{n+k}\right)=a_{k} ; \ldots \\
\left(T_{\rho ; P_{n+k}}^{(1)}\right)_{a_{k}}^{(m)}=\frac{m !}{\left(z-a_{k}\right)^{m+1}}+O(1), 1 \leq m \leq k_{n+k}\left(P_{n+k}\right)-1,
\end{gathered}
$$

where $k_{n+k}\left(P_{n+k}\right)$ is an order of a pole in the point $P_{n+k}$ for $f, \mathrm{k}=1, \ldots, \mathrm{s}$.

It follows that

Theorem 3.1. Let $f$ be a branch of the function of the class $M_{1}$ for essential character $\rho$ on a variable Riemann surface $F_{\mu}^{\prime}$ of type $(g, n), g \geq 2, n>0$, with pairwise different poles in $P_{n+1}, \ldots, P_{n+s}$ of the multiplicities $k_{n+1}, \ldots, k_{n+s}$ with given principal parts in them.

Then for the analytical continuation $f$ it is true that

$$
(f) \geq \frac{1}{P_{1}^{k_{1}} \ldots P_{n+s}^{k_{n+s}}}, k_{j} \geq 0, j=1, \ldots, n+s,
$$

on $F_{\mu}$ and

$$
f=\sum_{j=1}^{n+s} \sum_{m=1}^{k_{j}}\left[\frac{A_{j, m}}{(m-1) !} \frac{\partial^{m-1} T_{\rho ; P_{j}}^{(1)}}{\partial P_{j}^{m-1}}\right]+\sum_{j=1}^{g-1} \tilde{c}_{j} \int_{p_{0}}^{P} \tilde{\zeta}_{j},
$$

where

$f=\frac{A_{j, k_{j}}}{\left(z-z\left(P_{j}\right)\right)^{k_{j}}}+\ldots+\frac{A_{j, 2}}{\left(z-z\left(P_{j}\right)\right)^{2}}+\frac{A_{j, 1}}{\left(z-z\left(P_{j}\right)\right)}+O(1) \mathrm{f}$ or some branch in the neighborhood $P_{j}, j=1, \ldots, n+s$ on $F_{\mu}$, and all summands holomorphically depend on $[\mu]$ and $\rho$.

Now let $\rho$ be an inessential character. The proof of the preceding expansion formula for essential character is not applicable because in this case Prym integral of the second kind with a single simple pole on $F_{\mu}$ doesn't exist. That is Prym differential of the second kind for inessential character $\rho$ has to have at least two second-order poles in different arbitrary points $Q_{1}$ and $Q_{2}$ on $\Delta_{\mu}$, and with zero residues in $Q_{1}$ and $Q_{2}$. In this case Prym integrals $T_{\rho ; Q_{1}, Q_{2}}=-\int_{Q_{0}}^{P} \tau_{\rho ; Q_{1}^{2} Q_{2}^{2}}$ of the second kind with two simple poles $Q_{1}$ and $Q_{2}$ should be used as prime elements of expansion.

Let us consider another Prym differential $\tau_{\rho ; Q_{1} Q_{2}}=f_{0} \tau_{Q_{1} Q_{2}}$ of the third kind on $F_{\mu}$, where $f_{0}$ is a unit for $\rho$ on $F_{\mu}$ and $\tau_{Q_{1} Q_{2}}$ is a normalized Abelian differential with simple poles $Q_{1}$ and $Q_{2}$ on $F_{\mu}$, and residues +1 and -1 in these points accordingly, which holomorphically depend on $[\mu]$ and $\rho$. It has been known that $\tau_{Q_{1} Q_{2}}=d \Pi_{Q_{1} Q_{2}}$ and Abelian differential $\Pi_{Q_{1} Q_{2}}$ is expressed through the Riemann theta function for the surface $F_{\mu}$. In such case it is equal to the sum 
of two functions, one of which only depends on $Q_{1}$, and another only depends on $Q_{2}$ [2, p. 117]. Thus a derivative $\frac{\partial \Pi_{Q_{1} Q_{2}}}{\partial Q_{1}}$ doesn't depend on $Q_{2}$.

Prym differential $\tau_{\rho ; Q_{1}}^{(2)}$ has expansion $\left(\frac{1}{\left(z-z_{1}\right)^{2}}+\frac{c_{-1}^{(1)}}{\left(z-z_{1}\right)}+O(1)\right) d z$ in the neighborhood of the point $Q_{1}, z\left(Q_{1}\right)=z_{1}$, where $c_{-1}^{(1)}=\sum_{j=1}^{g} \log \rho\left(a_{j}\right) \varphi_{j}^{\prime}\left(Q_{1}\right)[6]$.

Prym differential $\tau_{\rho ; Q_{2}}^{(2)}$ also has expansion $\left(\frac{1}{\left(z-z_{2}\right)^{2}}+\frac{c_{-1}^{(2)}}{\left(z-z_{2}\right)}+O(1)\right) d z$ in the neighborhood of the point $\quad Q_{2}, z\left(Q_{2}\right)=z_{2}$, where $c_{-1}^{(2)}=\sum_{j=1}^{g} \log \rho\left(a_{j}\right) \varphi_{j}^{\prime}\left(Q_{2}\right)$.

A differential with two poles of the second order and zero residues in these points can be set in the following way

$$
\begin{aligned}
\tau_{\rho ; Q_{1}^{2} Q_{2}^{2}} & =c_{-1}^{(2)} f_{0}\left(Q_{1}\right) \tau_{\rho ; Q_{1}}^{(2)}-c_{-1}^{(1)} f_{0}\left(Q_{2}\right) \tau_{\rho ; Q_{2}}^{(2)}- \\
& -c_{-1}^{(1)} c_{-1}^{(2)} \tau_{\rho ; Q_{1} Q_{2}} .
\end{aligned}
$$

Let us denote that the principal part for $\tau_{\rho ; Q_{1} Q_{2}}$ in the point $Q_{1}$ takes the form of $\frac{f_{0}\left(Q_{1}\right)}{z-z_{1}}$ and in the point $Q_{2}$ takes the form of $\frac{f_{0}\left(Q_{2}\right)}{z-z_{2}}$. It follows that the constructed differential $\tau_{\rho ; Q_{1}^{2} Q_{2}^{2}}$ has two poles of the second order in $Q_{1}$ and $Q_{2}$, and two residues in this points. In the neighborhood of the point $Q_{1}$ its principal part takes the form of

$$
\begin{aligned}
& c_{-1}^{(2)} f_{0}\left(Q_{1}\right)\left[\frac{1}{\left(z-z_{1}\right)^{2}}+\frac{c_{-1}^{(1)}}{z-z_{1}}\right]- \\
& -c_{-1}^{(1)} c_{-1}^{(2)} \frac{f_{0}\left(Q_{1}\right)}{z-z_{1}}=\frac{c_{-1}^{(2)} f_{0}\left(Q_{1}\right)}{\left(z-z_{1}\right)^{2}}
\end{aligned}
$$

that is similar in the point $Q_{2}$

$$
\begin{aligned}
& c_{-1}^{(1)} f_{0}\left(Q_{2}\right)\left[\frac{1}{\left(z-z_{2}\right)^{2}}+\frac{c_{-1}^{(2)}}{z-z_{2}}\right]+ \\
& +c_{-1}^{(1)} c_{-1}^{(2)} \frac{f_{0}\left(Q_{2}\right)}{z-z_{2}}=-\frac{c_{-1}^{(1)} f_{0}\left(Q_{2}\right)}{\left(z-z_{2}\right)^{2}} .
\end{aligned}
$$

It is clear that a constructed differential $\tau_{\rho ; Q_{1}^{2} Q_{2}^{2}}$ holomorphically depends on $[\mu]$ and $\rho$.

From all has been said it follows that derivative $\frac{\partial T_{\rho ; Q_{1} Q_{2}}}{\partial Q_{1}}$ doesn't depend on $Q_{2}$.

Theorem 3.2. Let $f$ be a branch of the function of the class $M_{1}$ with inessential character $\rho$ and pairwise different poles $P_{n+1}, \ldots, P_{n+s}$ of the multiplicity $k_{n+1}, \ldots, k_{n+s}$ with set principal parts in them on a variable Riemann surface $F_{\mu}^{\prime}$ of type $(g, n), g \geq 2, n>0$. Then for the analytical continution $f$ on $F_{\mu}$ it is true that

and

$$
(f) \geq \frac{1}{P_{1}^{k_{1}} \ldots P_{n+s}^{k_{n+s}}}, k_{j} \geq 0, j=1, \ldots, n+s,
$$

$$
f(P)=\sum_{j=1}^{g} c_{j} \int_{P_{0}}^{P} f_{0} \zeta_{j}+\sum_{r=1}^{n+s-1} \frac{A_{r 1} T_{\rho ; P_{n+s} P_{r}}}{d_{n+s} f_{0}\left(P_{r}\right)}+
$$

$$
+\sum_{m=2}^{k_{1}} \frac{A_{1 m}}{(m-1) !} \frac{\partial^{m-1} T_{\rho ; P_{1} P_{n+1}}}{\partial P_{1}^{m-1}}+
$$

$+\sum_{j=2}^{n+s}\left[A_{j, 2} \frac{\partial T_{\rho ; P_{j} P_{1}}}{\partial P_{j}}+\frac{A_{j, 3}}{2 !} \frac{\partial^{2} T_{\rho ; P_{P} P_{1}}}{\partial P_{j}^{2}}+\ldots+\frac{A_{j, k_{j}}}{\left(k_{j}-1\right) !} \frac{\partial^{k_{j}-1} T_{\rho ; P_{j} P_{1}}}{\partial P_{j}^{k_{j}-1}}\right]+C$,

where

$$
f=\frac{A_{j, k_{j}}}{\left(z-z\left(P_{j}\right)\right)^{k_{j}}}+\ldots+\frac{A_{j, 2}}{\left(z-z\left(P_{j}\right)\right)^{2}}+\frac{A_{j, 1}}{z-z\left(P_{j}\right)}+O(1)
$$

for some branch in the neighborhood $P_{j}, j=1, \ldots, n+s$, on $F_{\mu}, C=0$ when $\rho \geq 1$,

$$
d_{k}=\sum_{m=1}^{g} \log \rho\left(a_{m}\right) \varphi_{m}^{\prime}\left(P_{k}\right), k=1, \ldots, n+s,
$$

on $F_{\mu}$, and all summands holomorphically depend on $[\mu]$ and $\rho$.

Proof. It suffices to verify a coincidence of principal parts on the left and right in this formula. For the neighborhood of the point $P_{r}, r=1, \ldots, n+s$, on $\Delta_{\mu}$ we have expansion in Laurent series

$$
\begin{aligned}
& \sum_{r=1}^{m+s-1}\left(\frac{d_{n+s} f_{0}\left(P_{r}\right)}{z-P_{r}}-\frac{d_{r} f_{0}\left(P_{n+s}\right)}{z-P_{n+s}}\right) \frac{A_{r 1}}{d_{n+s} f_{0}\left(P_{r}\right)}= \\
& =\frac{A_{r 1}}{z-P_{r}}+\ldots, r=1, \ldots, n+s-1 .
\end{aligned}
$$

For the neighborhood of the point $P_{n+s}$ we have expansion

$$
\begin{aligned}
& \sum_{r=1}^{n+s-1} \frac{-d_{r} f_{0}\left(P_{n+s}\right)}{z-P_{n+s}} \frac{A_{r 1}}{d_{n+s} f_{0}\left(P_{r}\right)}= \\
& =\frac{1}{z-P_{n+s}} \frac{f_{0}\left(P_{n+s}\right)}{d_{n+s}} \sum_{r=1}^{n+s-1} \frac{-d_{r} A_{r 1}}{f_{0}\left(P_{r}\right)}+\ldots=\frac{A_{n+s, 1}}{z-P_{n+s}}+\ldots,
\end{aligned}
$$

because

$$
\sum_{r=1}^{n+s} \frac{-A_{r 1} d_{r}}{f_{0}\left(P_{r}\right)}=0, \frac{f_{0}\left(P_{n+s}\right)}{d_{n+s}} \sum_{r=1}^{n+s-1} \frac{-d_{r} A_{r 1}}{f_{0}\left(P_{r}\right)}=A_{n+s, 1},
$$

according to the formula on full sum of residues for Abelian differentials $\frac{f}{f_{0}} d\left(\sum_{j=1}^{g} \log \rho\left(a_{j}\right) \varphi_{j}\right)$ of third kind on $F_{\mu}$, which in the point $P_{j}$ has residue $\frac{A_{j 1} d_{j}}{f_{0}\left(P_{j}\right)}, j=1, \ldots, n+s$. The theorem is proved.

Remark 3.1. P. Appell [2] has proven the theorem 3.2 for compact Riemann surface and simple poles. 
Every simple element (summand) depended on additional $g-1$ poles. In our work the theorem has been proved for a variable finite Riemann surface $F_{\mu}^{\prime}$ of genus $(g, n), g \geq 1, n>0$, and poles of any multiplicity. Moreover, every summand in our work has either one or two poles. Also when $\rho=1, n=0$ we obtain a classical fact about expansion of single-valued meromorphic function in sum of Abelian integrals on a compact Riemann surface.

\section{CONCLUSION}

Analogues of the residue theorem for Prym differentials of any entire order on variable finite Riemann surfaces are obtained for the first time. Thus three reciprocity laws have been proved. Analogues of P.Appell's expansion formula for functions with any characters on variable finite Riemann surfaces have been proved. In this case simple elements (summands) only have one or two poles.

\section{REFERENCES}

1. Dick R. Krichever - Novikov - like bases on punctured Riemann surfaces. Lett. Math. Phys, 1989, vol. 18, pp. 255-265,

2. Chueshev V.V. Mul'tiplikativnye funktsii i differentsialy Prima na peremennoy kompaktnoy rimanovoy poverkhnosti. Chast' 2 [Multiplicative functions and Prym differentials on a variable compact Riemann surface. Part 2]. Kemerovo: KemSU Publ., 2003. 248 p.

3. Al'fors L.V., Bers L. Prostranstva rimanovykh poverkhnostey i kvazikonformnye otobrazheniya [Spaces of Riemann surfaces and quasiconformal mappings]. Moscow: IL Publ., 1961. 177 p.

4. Farkas H.M., Kra I. Riemann surfaces. New-York: Springer-Verl., 1992. 363 p.

5. Earle C.J. Families of Riemann surfaces and Jacobi varieties. Annals of Mathematics, 1978, vol. 107, pp. 255-286.

6. Tulina M.I., Chueshev V.V. Differentsialy Prima na peremennoy kompaktnoy rimanovoy poverkhnosti [Prym Differentials on a variable compact Riemann surface]. Matematicheskie zametki [Math Zametki], 2014, vol. 95, no. 3, pp. 459-476.

7. Kazantseva A.A., Chueshev V.V. Differentsialy Prima na konechnoy rimanovoy poverkhnosti [Prym Differentials on a Finite Riemann surface]. Sibirskiy matematicheskiy zhurnal [Siberian Mathematical Journal], 2012, vol. 53, no. 1, pp. 89-106.

\section{Viktor V. Chueshev}

Dr.Sci.(Phys.-Math.), Professor, Professor of the Department of Fundamental Mathematics, Kemerovo State University, Kemerovo, Russian Federation.

\section{Aleksandr V. Chueshev}

Cand.Sci.(Phys.-Math.), Associate Professor, Associate Professor of the Department of Fundamental Mathematics, Kemerovo State University, Kemerovo, Russian Federation. 